\title{
Judicious partitions of bounded-degree graphs
}

\author{
B. Bollobás *† A.D. Scott ${ }^{\ddagger}$
}

\begin{abstract}
We prove results on partitioning graphs $G$ with bounded maximum degree. In particular, we provide optimal bounds for bipartitions $V(G)=V_{1} \cup V_{2}$ in which we minimize $\max \left\{e\left(V_{1}\right), e\left(V_{2}\right)\right\}$.
\end{abstract}

\section{Introduction}

The Max Cut problem asks for the maximum size of a cut in a graph $G$. By considering random cuts, it is easy to see that every graph with $m$ edges has a cut of size at least $m / 2$ (and the obvious greedy algorithm achieves this); sharper bounds for the extremal problem were obtained by Edwards ([10], [11]), who showed that every graph $G$ with $m$ edges has a bipartition $V(G)=V_{1} \cup V_{2}$ with

$$
e\left(V_{1}, V_{2}\right) \geq \frac{m}{2}+\sqrt{\frac{m}{8}+\frac{1}{64}}-\frac{1}{8}
$$

(For subsequent work, see [1],[2],[7], [20], and [13], [15].) Of course, maximizing $e\left(V_{1}, V_{2}\right)$ over partitions $V(G)=V_{1} \cup V_{2}$ is equivalent to minimizing $e\left(V_{1}\right)+e\left(V_{2}\right)$; here we shall be concerned with minimizing $\max \left\{e\left(V_{1}\right), e\left(V_{2}\right)\right\}$. Problems of this type, which involve finding a bipartition in which each

*Trinity College, Cambridge CB2 1TQ and Department of Mathematical Sciences, University of Memphis, Memphis TN38152; email: bollobas@msci.memphis.edu

${ }^{\dagger}$ Research supported in part by NSF grant ITR 0225610 and DARPA grant F3361501-C-1900

${ }^{\ddagger}$ Department of Mathematics, University College London, Gower Street, London WC1E 6BT; email: scott@math.ucl.ac.uk 
vertex class (or each subset of vertex classes) satisfies some condition simultaneously, are known as judicious partitioning problems (see [3], [4], [5], [6], $[7],[8])$.

The problem of finding a bipartition $V(G)=V_{1} \cup V_{2}$ minimizing $\max \left\{e\left(V_{1}\right), e\left(V_{2}\right)\right\}$ was addressed in [6] (see also [16], [17], [18]), where it was shown that every graph with $m$ edges has a bipartition in which each vertex class contains at most

$$
\frac{m}{4}+\sqrt{\frac{m}{32}+\frac{1}{256}}-\frac{1}{16}
$$

edges; indeed, there is a partition that satisfies both this bound and (1). It was also shown that there is a vertex partition into $r$ classes such that each vertex class contains at most

$$
\frac{m}{r^{2}}+\frac{r-1}{2 r^{2}}\left(\sqrt{2 m+\frac{1}{4}}-\frac{1}{2}\right)
$$

edges. These bounds are sharp for complete graphs on $r n+1$ vertices.

In this paper, we concentrate on graphs with bounded maximal degree. In section 2 we show that if $k \geq 3$ is odd then we can improve on (2) for graphs of maximal degree at most $k$ : every such graph has a bipartition in which each class contains at most

$$
\frac{k-1}{4 k} m+\frac{k-1}{4}
$$

edges; the extremal graphs are of the form $(2 t+1) K_{k} \cup s K_{k+1}$, for $s, t \geq 0$. As in [6], we can also demand that $e\left(V_{1}, V_{2}\right)$ is large: there is a bipartition satisfying (4) such that

$$
e\left(V_{1}, V_{2}\right) \geq \frac{k+1}{2 k} m .
$$

Note that (5) is sharp for graphs of the form $t K_{k} \cup s K_{k+1}$. We also show that stronger results hold for $k$-regular graphs.

In section 3 we discuss partitions in which we seek to bound the edges contained in each vertex class both from above and from below. For instance, given a graph with $m$ edges we would like a bipartition with close to $p^{2} m$ edges in one class and close to $(1-p)^{2} m$ in the other class. We prove a general result for partitions of oriented hypergraphs. 


\section{Bipartitions of bounded-degree graphs}

Our main result in this section is the following.

Theorem 1. Let $k \geq 3$ be an odd integer. Then every graph $G$ with $m$ edges and maximum degree at most $k$ has a vertex partition $V(G)=V_{1} \cup V_{2}$ such that, for $i=1,2$,

$$
e\left(V_{i}\right) \leq \frac{k-1}{4 k} m+\frac{k-1}{4}
$$

and

$$
e\left(V_{1}, V_{2}\right) \geq \frac{k+1}{2 k} m \text {. }
$$

The extremal graphs for (6) are of the form $(2 t+1) K_{k} \cup s K_{k+1}$, for $s, t \geq 0$.

Proof. Suppose the theorem is false, and let $G$ be a counterexample with a minimal number of vertices. Then either $G$ has no partition satisfying (6) and (7), or $G$ has no partition satisfying (6) with strict inequality and $G$ is not of the form $(2 t+1) K_{k} \cup s K_{k+1}$.

Note first that $G$ contains no component $C$ isomorphic to $K_{k+1}$, or applying the theorem to $G \backslash C$ yields the result for $G$ (note that $K_{k+1}$ can be partitioned into two vertex classes of size $(k+1) / 2)$. Thus Brooks Theorem implies that there is a proper colouring $c: V(G) \rightarrow[k]=\{1, \ldots, k\}$. Let $[k]=A \cup B$ be a random partition into sets $A, B$ with $|A|=(k+1) / 2$ and $|B|=(k-1) / 2$, chosen with equal probability among all such partitions. Then, writing $V_{S}=\{x: c(x) \in S\}$ for each $S \subset[k]$, we have

$$
\mathbb{E} e\left(V_{A}, V_{B}\right)=\frac{\frac{k+1}{2} \frac{k-1}{2}}{\left(\begin{array}{l}
k \\
2
\end{array}\right)} m=\frac{k+1}{2 k} m .
$$

Let $V(G)=V_{1} \cup V_{2}$ be a cut of $G$ with maximal size: then (8) implies $e\left(V_{1}, V_{2}\right) \geq \frac{k+1}{2 k} m$. We choose such a cut with $\max \left\{e\left(V_{1}\right), e\left(V_{2}\right)\right\}$ minimal. We may assume that $e\left(V_{1}\right) \geq e\left(V_{2}\right)$ Note that since $e\left(V_{1}, V_{2}\right)$ is maximal, we have $\left|\Gamma(v) \cap V_{2}\right| \geq\left|\Gamma(v) \cap V_{1}\right|$ for $v \in V_{1}$ and $\left|\Gamma(v) \cap V_{1}\right| \geq\left|\Gamma(v) \cap V_{2}\right|$ for $v \in V_{2}$, where $\Gamma(v)$ denotes the set of neighbours of $v$ (or else we could move $v$ to the opposite side and increase the size fo the cut).

Suppose

$$
e\left(V_{1}\right)=\frac{k-1}{4 k} m+\alpha
$$


SO

$$
\begin{aligned}
e\left(V_{2}\right) & \leq m-e\left(V_{1}, V_{2}\right)-e\left(V_{1}\right) \\
& \leq m-\frac{k+1}{2 k} m-\frac{k-1}{4 k} m-\alpha \\
& =\frac{k-1}{4 k} m-\alpha .
\end{aligned}
$$

If $\alpha<\frac{k-1}{4}$ the partition $V_{1} \cup V_{2}$ will do. Thus we may assume that $\alpha \geq \frac{k-1}{4}$.

If there is a vertex $v \in V_{1}$ with $\left|\Gamma(v) \cap V_{1}\right|=\left|\Gamma(v) \cap V_{2}\right|$ then $d(v) \leq k-1$ (since $k$ is odd): moving $v$ from $V_{1}$ to $V_{2}$ gives a partition $V_{1}^{\prime} \cup V_{2}^{\prime}$ with $e\left(V_{1}^{\prime}, V_{2}^{\prime}\right)=e\left(V_{1}, V_{2}\right)$ and $e\left(V_{1}^{\prime}\right)<e\left(V_{1}\right)$, while

$$
e\left(V_{2}^{\prime}\right) \leq e\left(V_{2}\right)+\frac{k-1}{2} \leq \frac{k-1}{4 k} m-\alpha+\frac{k-1}{2} .
$$

By minimality of $\max \left\{e\left(V_{1}\right), e\left(V_{2}\right)\right\}$, and since $e\left(V_{1}^{\prime}\right)<e\left(V_{1}\right)$, we must have $e\left(V_{2}^{\prime}\right) \geq e\left(V_{1}\right)$. Since $\alpha \geq(k-1) / 4$ it follows from (9) and (11) that $\alpha=$ $(k-1) / 4$, and we have equality in (8), (9) and (10), so there is a partition satisfying (6) and (7).

Otherwise, no $v \in V_{1}$ has $\left|\Gamma(v) \cap V_{1}\right|=\left|\Gamma(v) \cap V_{2}\right|:$ we shall show that then $G$ has a partition satisfying (6) and (7) strictly. Let $W_{1} \subset V_{1}$ be minimal such that $e\left(W_{1}\right) \geq e\left(V \backslash W_{1}\right)$ and $e\left(W_{1}\right) \geq \frac{k-1}{4 k} m+\frac{k-1}{4}$. Let $W_{2}=V \backslash W_{1}$; note that every $v \in W_{1}$ satisfies $\left|\Gamma(v) \cap W_{2}\right|>\left|\Gamma(v) \cap W_{1}\right|$. Let

$$
R=\min _{v \in W_{1}} \frac{\left|\Gamma(v) \cap W_{2}\right|}{\left|\Gamma(v) \cap W_{1}\right|} \geq \frac{k+1}{k-1} .
$$

We write

$$
e\left(W_{1}\right)=\frac{k-1}{4 k} m+\frac{k-1}{4}+\beta,
$$

where $\beta \geq 0$. Since $\left|\Gamma(v) \cap W_{2}\right| \geq R\left|\Gamma(v) \cap W_{1}\right|$ for $v \in W_{1}$, summing over $v \in W_{1}$ yields

$$
e\left(W_{1}, W_{2}\right) \geq 2 R e\left(W_{1}\right)=R \frac{k-1}{2 k} m+R \frac{k-1}{2}+2 \beta R,
$$

and so, by (12) and (13),

$$
e\left(W_{2}\right) \leq m-\left(\frac{k-1}{4 k}-R \frac{k-1}{2 k}\right) m-(2 R+1)\left(\frac{k-1}{4}+\beta\right) .
$$


Since $R \geq(k+1) /(k-1),(13)$ and (14) imply that

$$
e\left(W_{1}, W_{2}\right) \geq \frac{k+1}{2 k} m+R \frac{k-1}{2}
$$

and

$$
e\left(W_{2}\right) \leq \frac{k-1}{k} \frac{m}{4}-(2 R+1) \frac{k-1}{4} .
$$

Let $v \in W_{1}$ be a vertex with $\left|\Gamma(v) \cap W_{2}\right|=R\left|\Gamma(v) \cap W_{1}\right|$. Then let $X_{1}=$ $W_{1} \backslash\{v\}, X_{2}=W_{2} \cup\{v\}$. Clearly $e\left(X_{1}\right)<e\left(W_{1}\right)$ : we claim $e\left(X_{1}, X_{2}\right)>\frac{k+1}{2 k} m$ and $e\left(X_{2}\right)<\frac{k-1}{4 k} m+\frac{k-1}{4}$; by minimality of $W_{1}, e\left(X_{1}\right)<\frac{k-1}{4 k} m+\frac{k-1}{4}$. To prove the bound on $e\left(X_{2}\right)$, it is enough by (16) to show

$$
\frac{R}{1+R} d(v)<(2 R+2) \frac{k-1}{4}
$$

since $e\left(X_{2}\right)=e\left(W_{2}\right)+\left|\Gamma(v) \cap W_{2}\right|=e\left(W_{2}\right)+R d(v) /(1+R)$. Since $d(v) \leq k$, this follows if $2 R k<(k-1)(R+1)^{2}$, which holds since $R>1$ and $k \geq 3$. From (15) the bound on $e\left(X_{1}, X_{2}\right)$ holds if

$$
\frac{R-1}{R+1} k<R \frac{k-1}{2}
$$

since $e\left(X_{1}, X_{2}\right)=e\left(W_{1}, W_{2}\right)-\left|\Gamma(v) \cap W_{2}\right|+\left|\Gamma(v) \cap W_{1}\right|$. Rearranging, we see that this is equivalent to $2 /(k-1)<R+2 R /(R-1)$, which holds since $R>1$.

We have shown that $G$ has a partition satisfying (6) and (7). If $G$ has no partition satisfying (6) with strict inequality then we must have $\alpha=(k-1) / 4$ and equality in (9) and (10). So

$$
\begin{aligned}
e\left(V_{1}\right) & =\frac{k-1}{4 k} m+\frac{k-1}{4} \\
e\left(V_{2}\right) & =\frac{k-1}{4 k} m-\frac{k-1}{4}
\end{aligned}
$$

and so

$$
e\left(V_{1}, V_{2}\right)=\frac{k+1}{2 k} m .
$$

It follows that no cut of $G$ has size more than $\frac{k+1}{2 k} m$; in particular, every partition $V_{A} \cup V_{B}$ in (8) has size $\frac{k+1}{2 k} m$, and this must hold regardless of the $k$-colouring. It follows that no vertex $v$ of $G$ has degree less than $k-1$, or 
else there is a cut in (8) that would change size if we recoloured $v$. Similarly, no vertex $v$ has degree $k$ : otherwise, $v$ must have neighbours in every vertex class (or else we could change the size of some cut): letting $B \subseteq[k]$ consist of $(k-1) / 2$ colours in which $v$ has exactly one neighbour, and $A=[k] \backslash B$, the cut $V_{A} \cup V_{B}$ must have size $\frac{k+1}{2 k} m$, while the cut $V_{A} \backslash\{v\}, V_{B} \cup\{v\}$ is strictly larger. We deduce that $G$ is $(k-1)$-regular. Finally, if any component of $G$ is not isomorphic to $K_{k}$ then it can be $(k-1)$-coloured, which will yield a larger cut.

We remark that for $k$ even the theorem above (for $k+1$ ) immediately gives an optimal bound; the extremal graphs are of the form $(2 t+1) K_{k+1}$ for $t \geq 0$.

For $k$-regular graphs we can get a stronger result.

Theorem 2. Let $k \geq 3$ be an odd integer. Then every $k$-regular graph $G$ has a bipartition $V(G)=V_{1} \cup V_{2}$ such that $\left|V_{1}\right|=\left|V_{2}\right|$ and

$$
\max \left\{e\left(V_{1}\right), e\left(V_{2}\right)\right\} \leq \frac{k-1}{4 k} m .
$$

The extremal graphs are $s K_{k+1}$ for $s \geq 1$.

Proof. Consider a partition with $\left|V_{1}\right|=\left|V_{2}\right|$; suppose that $e\left(V_{1}, V_{2}\right)$ is maximal among such partitions. Note that since $G$ is regular we have $e\left(V_{1}\right)=$ $e\left(V_{2}\right)$. Thus we need only find a partition in which one vertex class satisfies (17). Now if $\left|\Gamma(v) \cap V_{2}\right|>\left|\Gamma(v) \cap V_{1}\right|$ for all $v \in V_{1}$ then

$$
e\left(V_{1}\right) \leq \frac{1}{2}\left|V_{1}\right| \frac{k-1}{2}=\frac{k-1}{8}|G|=\frac{k-1}{4 k} m,
$$

and similarly for $e\left(V_{2}\right)$ if $\left|\Gamma(v) \cap V_{1}\right|>\left|\Gamma(v) \cap V_{2}\right|$ for all $v \in V_{2}$. Otherwise, we can find $v \in V_{1}$ with $\left|\Gamma(v) \cap V_{1}\right|>\left|\Gamma(v) \cap V_{2}\right|$ and $w \in V_{2}$ with $\left|\Gamma(w) \cap V_{2}\right|>$ $\left|\Gamma(w) \cap V_{1}\right|$ : exchanging $v$ and $w$ gives a cut with larger size.

Now if (18) holds with equality then we may assume $\left|\Gamma(v) \cap V_{2}\right|>\mid \Gamma(v) \cap$ $V_{1} \mid$ for all $v \in V_{1}$, and in particular that every vertex in $V_{1}$ has $\left|\Gamma(v) \cap V_{2}\right|=$ $(k+1) / 2$. If there is $v \in V_{2}$ with $\left|\Gamma(v) \cap V_{1}\right|<\left|\Gamma(v) \cap V_{2}\right|$ then exchanging $v$ with any vertex adjacent to $v$ in $V_{1}$ gives a larger cut: we deduce that $\left|\Gamma(v) \cap V_{1}\right|>\left|\Gamma(v) \cap V_{2}\right|$ for all $v \in V_{2}$, and thus $\left|\Gamma(v) \cap V_{1}\right|=(k+1) / 2$ for all $v \in V_{2}$. Thus every vertex in $V_{1}$ and $V_{2}$ has exactly $(k+1) / 2$ neighbours in the opposite class, and furthermore this holds for every bipartition into sets of equal size satisfying (18). If $v \in V_{1}$ and $w \in V_{2}$ are adjacent then 
exchanging $v$ and $w$ must leave every vertex with $(k+1) / 2$ neighbours in the opposite class, and so we must have $\Gamma(v) \cup\{v\}=\Gamma(w) \cup\{w\}$. It is then easy to check that every component of $G$ must be isomorphic to $K_{k+1}$.

A similar result to Theorem 2 holds when $k$ is even.

Theorem 3. Let $k \geq 2$ be an even integer. Then every $k$-regular graph $G$ with even order has a bipartition $V(G)=V_{1} \cup V_{2}$ with $\left|V_{1}\right|=\left|V_{2}\right|$ and

$$
\max \left\{e\left(V_{1}\right), e\left(V_{2}\right)\right\} \leq \frac{1}{4} \frac{k}{k+1} m .
$$

The extremal graphs are of the form $2 t K_{k+1}, t \geq 1$.

Every $k$-regular graph of odd order has a bipartition with $\left|V_{1}\right|=\left|V_{2}\right|-1$ and

$$
\max \left\{e\left(V_{1}\right), e\left(V_{2}\right)\right\} \leq \frac{1}{4} \frac{k}{k+1} m+\frac{k}{4} .
$$

The extremal graphs are of the form $(2 t+1) K_{k+1}, t \geq 0$.

Proof. If $|G|$ is even then let $V(G)=V_{1} \cup V_{2}$ be a partition with $\left|V_{1}\right|=$ $\left|V_{2}\right|$, and $e\left(V_{1}, V_{2}\right)$ maximal among such partitions. If there is $v \in V_{1}$ with $\left|\Gamma(v) \cap V_{2}\right|>\left|\Gamma(v) \cap V_{1}\right|$ and $w \in V_{2}$ with $\left|\Gamma(w) \cap V_{1}\right| \geq\left|\Gamma(w) \cap V_{2}\right|$ then exchanging $v$ and $w$ gives a bigger cut; similarly, if there is $v \in V_{1}$ with $\left|\Gamma(v) \cap V_{1}\right| \geq\left|\Gamma(w) \cap V_{2}\right|$ and $w \in V_{2}$ with $\left|\Gamma(w) \cap V_{1}\right|>\left|\Gamma(w) \cap V_{2}\right|$ we can exchange $v$ and $w$. Also, if there are adjacent vertices $v \in V_{1}$ and $w \in V_{2}$ with $\left|\Gamma(v) \cap V_{1}\right|=\left|\Gamma(v) \cap V_{2}\right|$ and $\left|\Gamma(w) \cap V_{1}\right|=\left|\Gamma(w) \cap V_{2}\right|$ then exchanging $v$ and $w$ increases the size of the cut.

For a maximal cut, if $\left|\Gamma(v) \cap V_{2}\right|>\left|\Gamma(v) \cap V_{1}\right|$ for all $v \in V_{1}$, then summing degrees in $V_{1}$ gives

$$
e\left(V_{1}\right) \leq \frac{1}{2}\left|V_{1}\right| \frac{k-2}{2}=\frac{k-2}{4 k} m<\frac{1}{4} \frac{k}{k+1} m,
$$

and similarly for $e\left(V_{2}\right)$ if $\left|\Gamma(v) \cap V_{1}\right|>\left|\Gamma(v) \cap V_{2}\right|$ for all $v \in V_{2}$. Since $e\left(V_{1}\right)=e\left(V_{2}\right)$, this proves the bound in these cases.

Otherwise, let $S_{i}=\left\{v \in V_{i}:\left|\Gamma(v) \cap V_{2}\right|=\left|\Gamma(v) \cap V_{1}\right|\right\}$, for $i=1,2$. Note that there are no edges between $S_{1}$ and $S_{2}$, or we can obtain a larger cut by exchanging adjacent vertices in $S_{1}$ and $S_{2}$. Let $T_{i}=V_{i} \backslash S_{i}$ for $i=1,2$. Now,

$$
e\left(S_{1}, V_{2}\right)=\sum_{v \in S_{1}}\left|\Gamma(v) \cap V_{2}\right|=\sum_{v \in S_{1}}\left|\Gamma(v) \cap V_{1}\right|
$$


and

$$
e\left(S_{2}, V_{1}\right)=\sum_{v \in S_{2}}\left|\Gamma(v) \cap V_{1}\right|=\sum_{v \in S_{2}}\left|\Gamma(v) \cap V_{2}\right| .
$$

Since $\left|\Gamma(v) \cap V_{2}\right| \geq(k+2) / 2$ and $\left|\Gamma(v) \cap V_{1}\right| \leq(k-2) / 2$ for $v \in T_{1}$, we have

$$
\sum_{v \in T_{1}}\left|\Gamma(v) \cap V_{1}\right| \leq \frac{k-2}{k+2} \sum_{v \in T_{1}}\left|\Gamma(v) \cap V_{2}\right|=\frac{k-2}{k+2} e\left(T_{1}, V_{2}\right) .
$$

Now

$$
\begin{aligned}
2 e\left(V_{1}\right) & =\sum_{v \in T_{1}}\left|\Gamma(v) \cap V_{1}\right|+\sum_{v \in S_{1}}\left|\Gamma(v) \cap V_{1}\right| \\
& \leq \frac{k-2}{k+2} e\left(T_{1}, V_{2}\right)+e\left(S_{1}, V_{2}\right) \\
& =\frac{k-2}{k+2} e\left(V_{1}, V_{2}\right)+\frac{4}{k+2} e\left(S_{1}, V_{2}\right)
\end{aligned}
$$

and similarly,

$$
2 e\left(V_{2}\right) \leq \frac{k-2}{k+2} e\left(V_{1}, V_{2}\right)+\frac{4}{k+2} e\left(S_{2}, V_{1}\right) .
$$

Since $e\left(S_{1}, S_{2}\right)=0$, we have $e\left(S_{1}, V_{2}\right)+e\left(S_{2}, V_{1}\right) \leq e\left(V_{1}, V_{2}\right)$, and so (20) and (21) imply

$$
\begin{aligned}
e\left(V_{1}\right)+e\left(V_{2}\right) & \leq \frac{k-2}{k+2} e\left(V_{1}, V_{2}\right)+\frac{2}{k+2}\left(e\left(S_{1}, V_{2}\right)+e\left(S_{2}, V_{1}\right)\right) \\
& \leq \frac{k}{k+2} e\left(V_{1}, V_{2}\right) \\
& =\frac{k}{k+2} e(G)-\frac{k}{k+2}\left(e\left(V_{1}\right)+e\left(V_{2}\right)\right) .
\end{aligned}
$$

Thus

$$
e\left(V_{1}\right)+e\left(V_{2}\right) \leq \frac{1}{2} \frac{k}{k+1} e(G)
$$

and since $e\left(V_{1}\right)=e\left(V_{2}\right),(22)$ implies that, for $i=1,2$,

$$
e\left(V_{i}\right) \leq \frac{1}{4} \frac{k}{k+1} e(G)
$$

Now if (23) holds with equality then we have equality in (20) and (21), so $\left|\Gamma(v) \cap V_{2}\right|=(k+2) / 2$ for $v \in T_{1}$ and $\left|\Gamma(v) \cap V_{1}\right|=(k+2) / 2$ for $v \in T_{2}$. 
We also have equality in $(22)$, so $e\left(S_{1}, V_{2}\right)+e\left(S_{2}, V_{1}\right)=e\left(V_{1}, V_{2}\right)$, and hence $e\left(T_{1}, T_{2}\right)=0$. Furthermore, this must hold for every partition with $\left|V_{1}\right|=\left|V_{2}\right|$ that satisfies (23). Now if a vertex $v \in S_{1}$ is adjacent to $w \in T_{1}$ then pick a vertex $x \in \Gamma(v) \cap V_{2}$, so $x \in T_{2}$. Exchanging $v$ and $x$ does not change the size of the cut, so $x$ must also be adjacent to $w$ (or else exchanging $v$ and $x$ would leave $w$ with more than $(k+2) / 2$ neighbours on the other side of the partition). But $x \in T_{2}$, so $x$ has no neighbours in $T_{1}$, hence we must have $e\left(S_{1}, T_{1}\right)=0$. In particular, we see that every component of $G\left[V_{1}\right]$ and $G\left[V_{2}\right]$ must be a regular graph. Finally, if $v \in V_{1}$ is adjacent to $w \in V_{2}$ then either $v \in S_{1}$ and $w \in T_{2}$ or $v \in T_{1}$ and $w \in S_{2}$, so exchanging $v$ and $w$ does not change the size of the cut. Without loss of generality, we may assume $v \in S_{1}$ and $w \in T_{2}$, and let $V_{1}^{\prime} \cup V_{2}^{\prime}$ be the resulting bipartition: then every component of $G\left[V_{1}^{\prime}\right]$ and $G\left[V_{2}^{\prime}\right]$ is regular. Since $\left|\Gamma(w) \cap V_{1}^{\prime}\right|=k / 2$ and $\left|\Gamma(v) \cap V_{2}^{\prime}\right|=(k-2) / 2$ (and $\Gamma(w) \cap V_{1}^{\prime} \subset S_{1}$ and $\left.\Gamma(v) \cap V_{2}^{\prime} \subset T_{2}\right)$, it follows that $\Gamma(v) \cup\{v\}=\Gamma(w) \cup\{w\}$. It follows that all components of $G$ are copies of $K_{k+1}$.

If $|G|$ is odd, say $|G|=2 l+1$, then consider partitions $V_{1} \cup V_{2}$ with $\left|V_{1}\right|=l$ and $\left|V_{2}\right|=l+1$. Note that $e(G)=(2 l+1) k / 2$ and $e\left(V_{2}\right)=$ $e\left(V_{1}\right)+k / 2$. Applying the same argument as above, we get three alternatives. If $\left|\Gamma(v) \cap V_{2}\right|>\left|\Gamma(v) \cap V_{1}\right|$ for all $v \in V_{1}$, we obtain

$$
e\left(V_{1}\right) \leq \frac{1}{2} l \frac{k-2}{2}=\frac{k-2}{4 k} m-\frac{k-2}{8}
$$

and so, since $m \leq\left(\begin{array}{c}k+1 \\ 2\end{array}\right)$,

$$
e\left(V_{2}\right)=e\left(V_{1}\right)+\frac{k}{2} \leq \frac{k-2}{4 k} m+\frac{3 k+2}{8} \leq \frac{1}{4} \frac{k}{k+1} m+\frac{k}{4},
$$

with equality only if $m=\left(\begin{array}{c}k+1 \\ 2\end{array}\right)$ and hence $G \equiv K_{k+1}$.

Alternatively, $\left|\Gamma(v) \cap V_{1}\right|>\left|\Gamma(v) \cap V_{2}\right|$ for all $v \in V_{2}$, and so

$$
e\left(V_{2}\right) \leq \frac{1}{2}(l+1) \frac{k-2}{2}=\frac{k-2}{4 k} m+\frac{k-2}{8}<\frac{1}{4} \frac{k}{k+1} m+\frac{k}{4} .
$$

Otherwise, define $S_{i}$ and $T_{i}$ as in the even case; the argument runs in the same way as in the even case, except $e\left(V_{2}\right)=e\left(V_{1}\right)+k / 2=\frac{1}{2}\left(e\left(V_{1}\right)+\right.$ $\left.e\left(V_{2}\right)\right)+k / 4$, so (23) becomes

$$
e\left(V_{i}\right) \leq \frac{1}{4} \frac{k}{k+1} m+\frac{k}{4}
$$

The argument for extremal graphs is identical. 
We remark that, in the case $k=3$, a stronger result than Theorem 2 follows from a result of Locke [14], who showed that every cubic $K_{4}$-free graph $G$ has a partition $V(G)=V_{1} \cup V_{2}$ with $\left|V_{1}\right|=\left|V_{2}\right|$ and $e\left(V_{1}, V_{2}\right) \geq$ $11 e(G) / 15$; since $e\left(V_{1}\right)=e\left(V_{2}\right)$ for a partition into classes of equal size, we have $\max \left\{e\left(V_{1}\right), e\left(V_{2}\right)\right\} \leq 2 e(G) / 15$. It would be interesting to determine the optimal constants for $k$-regular graphs containing no $K_{k+1}$. For graphs with large girth it should be possible to get even stronger results (see [9], [12], [14], [19], [8]).

We remark that a random $k$-regular graph, or a random graph in $\mathcal{G}(n, p)$ with $p=O(1 / n)$, contains (with high probability) only a few short cycles. What are the best constants we can get in the theorems above for random graphs? We shall consider this question elsewhere.

\section{Judicious partitions of hypergraphs}

In section 2 we showed that every graph $G$ with maximal degree bounded by a constant has a partition $V(G)=V_{1} \cup V_{2}$ in which $\max \left\{e\left(V_{1}\right), e\left(V_{2}\right)\right\}$ is not very large. However, this does not give us any information about $\min \left\{e\left(V_{1}\right), e\left(V_{2}\right)\right\}$. In a random bipartition, we have $\mathbb{E} e\left(V_{1}\right)=\mathbb{E} e\left(V_{2}\right)=m / 4$ and $\mathbb{E} e\left(V_{1}, V_{2}\right)=m / 2$ : in this section we show that, for bounded-degree graphs, we can get quite close to this. More generally, we prove a result for imbalanced partitions into $k \geq 2$ sets.

Theorem 4. For every integer $D \geq 1$ there is a constant $K$ such that for every graph $G$ with maximum degree at most $D$ and every sequence of nonnegative real numbers $p_{1}, \ldots, p_{k}$ with $\sum_{i=1}^{k} p_{i}=1$ there is a partition $V(G)=\bigcup_{i=1}^{k} V_{i}$ with, for $1 \leq i \leq k$,

$$
\left|e\left(V_{i}\right)-p_{i}^{2} e(G)\right| \leq K
$$

and, for $1 \leq i<j \leq k$,

$$
\left|e\left(V_{i}, V_{j}\right)-2 p_{i} p_{j} e(G)\right| \leq K .
$$

Furthermore, we may also demand ||$V_{i}\left|-p_{i}\right| G||<K$, for $1 \leq i \leq k$.

Theorem 4 is a special case of a rather general result about partitioning oriented hypergraphs. 
An oriented hypergraph $H$ is given by a set $V$ and a collection $E(H)$ of ordered tuples of (distinct) elements of $V$. For instance, if all tuples have size 2 then we obtain a digraph (note that we allow tuples of different sizes). Given oriented hypergraphs $H_{1}, \ldots, H_{s}$ with common vertex set $V$, sets $V_{1}, \ldots, V_{t} \subset V$, and integers $1 \leq k_{1}, \ldots, k_{u} \leq t$, we define

$$
d_{k_{1}, \ldots, k_{u}}^{(i)}\left(V_{1}, \ldots, V_{t}\right)=\left\{\left\langle v_{1}, \ldots, v_{u}\right\rangle \in E\left(H_{i}\right): v_{i} \in V_{k_{i}} \forall i\right\} .
$$

We write $e_{t}\left(H_{i}\right)$ for the number of edges with $t$ vertices (ie the number of $t$-tuples) in $E(H)$.

Theorem 5. For every triple $r, s, D$ of positive integers there is a constant $K=K(r, s, D)$ such that the following assertion holds for every $k \geq 1$. For every sequence of hypergraphs $H_{1}, \ldots, H_{s}$ with common vertex set $V$ such that each $H_{i}$ has maximum edge size at most $r$ and maximum vertex degree at most $D$, and every sequence of nonnegative reals $p_{1}, \ldots, p_{k}$ with $\sum_{i=1}^{k} p_{i}=1$, there is a partition $V=\bigcup_{i=1}^{k} V_{i}$ such that

$$
|| V_{i}\left|-p_{i}\right| V|| \leq K
$$

for $1 \leq i \leq k$, and, for $1 \leq i \leq k, 1 \leq t \leq r$, and $1 \leq k_{1}, \ldots, k_{t} \leq k$,

$$
\left|d_{k_{1}, \ldots, k_{t}}^{(i)}\left(V_{1}, \ldots, V_{k}\right)-e_{t}\left(H_{i}\right) \prod_{i=1}^{t} p_{i}\right|<K .
$$

We shall need the following lemma in the proof of Theorem 5 .

Lemma 6. Let $t, D \geq 1$ be integers. There is a constant $K=K(t, D)$ such that for every finite set $S$, every sequence $\left(f_{i}\right)_{i=1}^{t}$ of functions from $S$ to $\{0, \ldots, D\}$ and every positive integer $u$ and nonnegative reals $p_{1}, \ldots, p_{u}$ with $\sum_{i=1}^{u} p_{i}=1$, there is a partition $S=\bigcup_{i=1}^{u} S_{i}$ such that, for $1 \leq i \leq u$,

$$
|| S_{i}\left|-p_{i}\right| S||<K
$$

and, for $1 \leq i \leq u$ and $1 \leq j \leq t$,

$$
\left|\sum_{x \in S_{i}} f_{j}(x)-p_{i} \sum_{x \in S} f_{j}(x)\right|<K .
$$


Proof. Let $K=(D+1)^{t+1}$. Define an equivalence relation on $S$ by setting $x \sim$ $y$ if $f_{i}(x)=f_{i}(y)$ for $i=1, \ldots, t$. Let the equivalence classes be $R_{1}, \ldots, R_{T}$, where $T \leq(D+1)^{t}$. For $1 \leq l \leq T$, let $R_{l}=\bigcup_{i=1}^{u} Q_{l}^{(i)}$ be an arbitrary partition with $\left|Q_{l}^{(i)}\right|=\left\lfloor p_{i}\left|R_{j}\right|\right\rfloor$ or $\left|Q_{l}^{(i)}\right|=\left\lceil p_{i}\left|R_{j}\right|\right\rceil$ for each $i$. Note that ||$Q_{l}^{(i)}\left|-p_{i}\right| R_{l}|| \leq 1$. Now let $S_{i}=\bigcup_{l=1}^{T} Q_{l}^{(i)}$ for each $i$. Note that ||$S_{i} \mid-$ $p_{i}|V| \mid<T$. An easy calculation shows that, for $1 \leq i \leq u$ and $1 \leq j \leq t$,

$$
\begin{aligned}
\left|\sum_{x \in S_{i}} f_{j}(x)-p_{i} \sum_{x \in S} f_{j}(x)\right| & \leq \sum_{l=1}^{T}\left|\sum_{x \in Q_{l}^{(i)}} f_{j}(x)-p_{i} \sum_{x \in R_{l}} f_{j}(x)\right| \\
& =\sum_{l=1}^{T}\left|y_{l}\right|\left|Q_{l}^{(i)}\right|-p_{i}\left|R_{l}\right| \mid \\
& \leq D T \\
& <K
\end{aligned}
$$

wheer $y_{l}$ is the common value of $f_{j}(x)$ for $x \in R_{l}$.

We can now prove the theorem.

Proof of Theorem 5. We begin by considering the graph $H^{*}$ with vertex set $V$ and edges all pairs $\{x, y\}$ that are contained in some edge in some $H_{i}$. Note that $H^{*}$ has maximal degree less than $r s D$, so there is some partition $V=\bigcup_{i=1}^{u} V_{i}$ with $u \leq r s D$ such that each $V_{i}$ is independent in $H^{*}$.

We shall obtain the desired partition of $V$ by applying the lemma to each $V_{i}$ in turn. Suppose we wish to partition $V_{i}$, and we have partitioned $V_{j}$ as $\bigcup_{i=1}^{k} V_{j}^{(i)}$ for each $j<i$. We define an equivalence relation on the edges of $H_{1}, \ldots, H_{s}$ by setting edges $\left\langle v_{1}, \ldots, v_{a}\right\rangle$ and $\left\langle w_{1}, \ldots, w_{b}\right\rangle$ to be equivalent if (1) they belong to the same oriented hypergraph $H_{t}$ and (2) $a=b$ and (3) $v_{j}$ and $w_{j}$ are in the same vertex class $V_{l}$ for each $j$ and (4) if $l<i$ and $v_{j}, w_{j} \in V_{l}$ then $v_{j}$ and $w_{j}$ are in the same vertex class $V_{l}^{(h)}$. For each equivalence class $X$ we define a function $f_{X}$ on $V_{i}$ by setting $f_{X}(v)$ to be the number of edges in $X$ that contain $v$. Finally, we apply the lemma to this collection of functions.

An easy check shows that this yields the desired partition. (Note that for $1 \leq a \leq r$ and (distinct) $k_{1}, \ldots, k_{a} \in[u]$, the set of edges $\left\langle v_{1}, \ldots, v_{a}\right\rangle$ with $v_{i} \in V_{k_{i}}$ for $1 \leq i \leq a$ is essentially partitioned one vertex class $V_{k_{i}}$ at a time.) 
As an application of the theorem, we obtain the following result. For a graph $G$, a partition $V(G)=\bigcup_{i=1}^{k} V_{i}$, and integers $1 \leq k_{0}, \ldots, k_{t} \leq k$, let $P_{k_{0}, \ldots, k_{t}}$ be the number of paths $v_{0} \cdots v_{t}$ with $v_{i} \in V_{k_{i}}$ for each $i$. Let $P_{t}(G)$ be the number of paths of length $t$ in $G$.

Theorem 7. For every $T, D \geq 1$ there is a constant $K=K(T, D)$ such that, for $k \geq 1$, every graph $G$ with maximum degree at most $D$ has a partition $V(G)=\bigcup_{i=1}^{k} V_{i}$ such that, for every sequence $k_{0}, \ldots, k_{t}$ with $t \leq T$,

$$
\left|P_{k_{1}, \ldots, k_{t}}(G)-2 P_{t}(G) / k^{t}\right| \leq K .
$$

Similar results follow for embeddings of other subgraphs, and for imbalanced partitions.

\section{Conclusion}

In Theorem 5, we gave a result concerning simultaneous partitions of several hypergraphs with bounded degrees and the same vertex set. There are many related problems: for instance, what is the correct analogue of Theorem 1 for simultaneous bipartitions of more than one graph with the same vertex set and maximum degree at most $k$ ? A very natural question is whether bounds similar to (1) and (2) can be proved for simultaneous bipartitions of two graphs.

Problem 8. Find the largest integer $f^{(2)}(m)$ such that for every pair of graphs $G_{1}, G_{2}$ with $m$ edges and common vertex set $V$ there is a bipartition $V=V_{1} \cup V_{2}$ with

$$
\min \left\{e_{G_{1}}\left(V_{1}, V_{2}\right), e_{G_{2}}\left(V_{1}, V_{2}\right)\right\} \geq f^{(2)}(m) .
$$

Perhaps it is even possible to find a bipartition that gives a cut of size at least $(1+o(1)) m / 2$ in each graph. Note that in a random partition we have $\mathbb{E} e_{G_{1}}\left(V_{1}, V_{2}\right)=e\left(G_{1}\right) / 2$ and $\mathbb{E} e_{G_{2}}\left(V_{1}, V_{2}\right)=e\left(G_{2}\right) / 2$. But these two quantities are not independent, so we face a similar problem to judicious partitions, where we seek to maximize more than one quantity simultaneously. There are many possible extensions and related problems. For instance, what about simultaneous bisections, or cuts into more than two vertex classes? 


\section{References}

[1] N. Alon, Bipartite subgraphs, Combinatorica 16 (1996), 301-311

[2] N. Alon and E. Halperin, Bipartite subgraphs of integer-weighted graphs, Discrete Math. 181 (1998), 19-29

[3] B. Bollobás and A.D. Scott, Judicious partitions of graphs, Periodica Math. Hungar. 26 (1993) 127-139

[4] B. Bollobás and A.D. Scott, Judicious partitions of hypergraphs, J. Comb. Theory Ser. A 78 (1997) 15-31

[5] B. Bollobás and A.D. Scott, Judicious partitions of 3-uniform hypergraphs, European J. Comb., 21 (2000), 289-300

[6] B. Bollobás and A.D. Scott, Exact bounds for judicious partitions, Combinatorica 19 (1999), 473-486

[7] B. Bollobás and A.D. Scott, Better bounds for Max Cut, to appear

[8] B. Bollobás and A.D. Scott, Problems on judicious partitions, to appear

[9] J.A. Bondy and S.C. Locke, Largest bipartite subgraphs in triangle-free graphs with maximum degree three, J. Graph Theory 10 (1986), 477-504

[10] C.S. Edwards, Some extremal properties of bipartite subgraphs, Canadian J. Math. 25 (1973), 475-485

[11] C.S. Edwards, An improved lower bound for the number of edges in a largest bipartite subgraph, in Proc 2nd Czech. Symposium on Graph Theory, Prague (1975), 167-181

[12] G. Hopkins and W. Staton, Extremal bipartite subgraphs of cubic triangle-free graphs, J. Graph Theory 6 (1982), 115-121

[13] M. Laurent, Annotated Bibliographies in Combinatorial Optimization, M. Dell'Amico, F. Maffioli and S. Martello, eds, Wiley, 1997, 241-259

[14] S.C. Locke, Maximum k-colorable subgraphs, J. Graph Theory 6 (1982), $123-132$ 
[15] S. Poljak and Zs. Tuza, Maximum cuts and largest bipartite subgraphs, in Combinatorial Optimization, DIMACS Ser. in Discrete Mathematics and Theoretical Computer Science, volume 20, W. Cook, L. Lovász and P. Seymour, eds, xi+441pp, 181-244

[16] T.D. Porter, On a bottleneck bipartition conjecture of Erdős, Combinatorica 12 (1992), 317-321

[17] T.D. Porter, Graph partitions, J. Combin. Math. Combin. Comp. 15 (1994), 111-118

[18] T.D. Porter, Minimal partitions of a graph, Ars Combinatoria 53 (1999), 181-186

[19] W. Staton, Edge deletions and the chromatic number, Ars Combinatoria 10 (1980), 103-106

[20] Zs. Tuza, Maximum cuts: improvements and local algorithmic analogues of the Edwards-Erdős inequality, Discrete Math. 194 (1999), 39-58 